\title{
Relaciones entre topografía del terreno y morfología de los edificios volcánicos basálticos monogénicos de Tenerife (Islas Canarias, España)
}

\section{Relationship between topography and morphology of cinder cones of Tenerife (Canary Islands, Spain)}

\author{
Javier Dóniz Páez*
}

\section{INTRODUCCIÓN}

Los volcanes basálticos monogénicos (cinder cones, scoria cones) constituyen las formas eruptivas más comunes en el planeta (Wood, 1980a) (figura 1), se elaboran durante un sola fase eruptiva, involucran un tipo de magma (Cas y Wright, 1987), poseen un sistema de conductos simple que es el empleado durante esa única fase, que puede durar desde varios días a varios años y están asociados a ambientes tectónicos de extensión (Takada, 1994). En general, se trata de conos de piroclastos formados por la acumulación de depósitos de caída soldados o no, con formas simétricas e irregulares y de diferente tamaño (lapilli, bombas, bloques, escorias, depósitos de spatter, cenizas, etc.), xenolitos y lavas interestratificadas (Dóniz Páez et al., 2008). Los piroclastos se disponen formando capas de diferente potencia que varían desde varios centímetros hasta pocos metros (Vespermann y Schmincke, 2000; Calvari y Pinkerton, 2004; Martin y Németh, 2006; Bertotto et al., 2006; Valentine y Keating, 2007; Valentine et al., 2007).

* Escuela Universitaria de Turismo Iriarte, adscrita a la Universidad de la Laguna. España (jdoniz@ull.es).

El autor desea agradecer a los dos evaluadores anónimos por sus acertadas correcciones, comentarios y sugerencias sobre el trabajo, que sin duda han contribuido a mejorarlo y a enriquecerlo. 
Las características morfológicas generales de este tipo de volcanes han sido estudiadas, descritas y expuestas tanto en manuales clásicos sobre volcanología (Rittmann, 1963; Macdonald, 1972; Cas y Wright, 1987; Ollier, 1988; etc.), como en trabajos mucho más específicos (Wood, 1980a y b; Romero, 1991 y 1992; Poblete, 1994; Pallí y Pujadas, 1999; González-Cárdenas et al., 2006; Becerra-Ramírez, 2007; Dóniz Páez, 2009; etc.). Unos y otros resaltan las diferencias morfoestructurales con las que son analizados los volcanes basálticos monogénicos. De este modo, en los trabajos generales son definidos como estructuras eruptivas muy simples, mientras que en las publicaciones específicas se resalta su heterogeneidad volcanomorfológica.

En este sentido, tradicionalmente solo se hace referencia a dos categorías morfológicas: los conos anulares cerrados y los conos abiertos o en herradura, que constituyen las morfologías más habituales. Sería conveniente señalar que la idea simplificada de este tipo de formas volcánicas descansa, en muchas ocasiones, en la consideración de que los conos volcánicos tienen, en origen, una morfología anular, de modo que todos aquellos edificios eruptivos que no poseen esta forma corresponden a erupciones en las que ha existido algún tipo de perturbación ajena al proceso estrictamente volcánico (acción del viento, pendiente, etc.). No obstante, es evidente para cualquier investigador que trabaje en geomorfología volcánica que los aparatos eruptivos pueden adquirir morfologías no siempre tan sencillas sin que existan estos factores perturbadores.

FIGURA 1

VOLCANES BASÁLTICOS MONOGÉNICOS DE TENERIFE CORRESPONDIENTES A LAS ERUPCIONES DE GARACHICO DE 1706 (IZQUIERDA) Y DE EL CHINYERO DE 1909 (DERECHA)
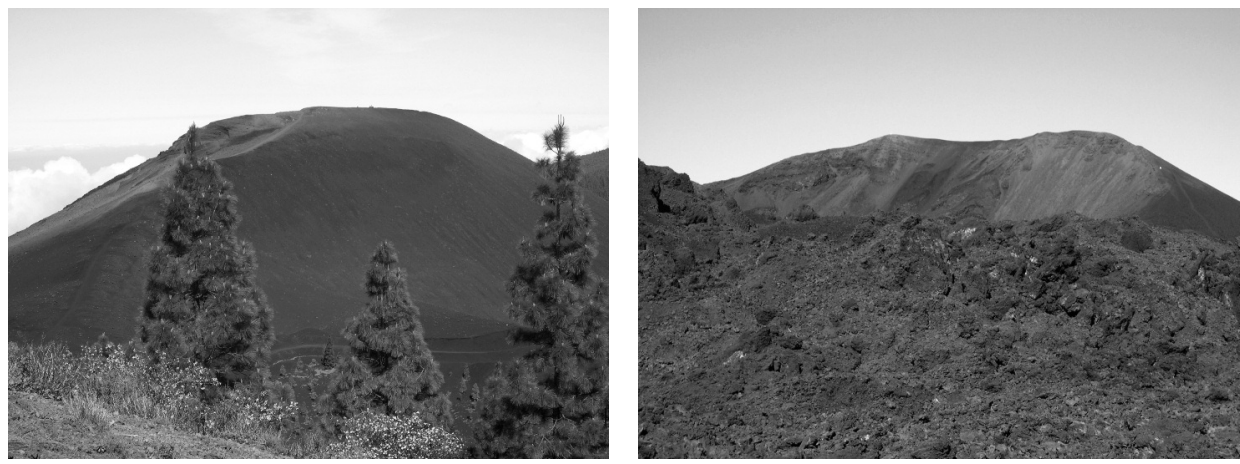

Estudios Geográficos, Vol. LXXII, 270, pp. 59-75, enero-junio 2011 ISSN: 0014-1496, eISSN: 1988-8546, doi: 10.3989/estgeogr.201103 


\section{FIGURA 2}

BLOQUE DIAGRAMA QUE MUESTRA EL CONTROL QUE EJERCE LA PENDIENTE EN LA APERTURA DE LOS CRÁTERES Y EN LA MORFOLOGÍA DEL VOLCÁN (DÓNIZ PÁEZ, 2001)

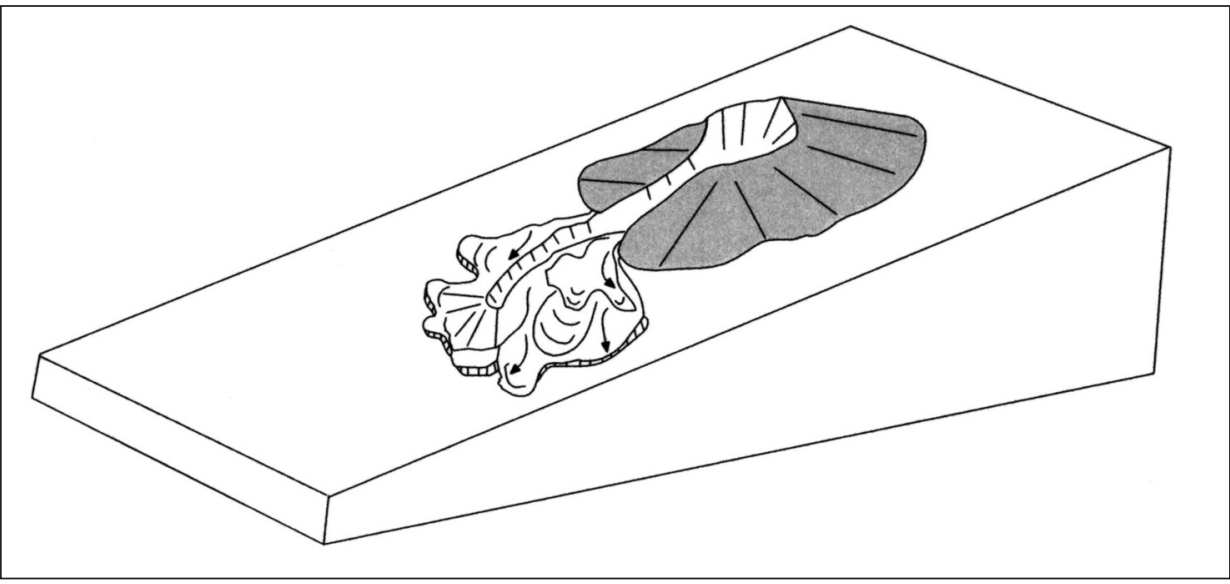

Una de las características más diferenciadoras de este tipo de volcanes es si poseen o no cráteres y, cuando los tienen, si están abiertos o cerrados. Uno de los aspectos sobre los que la topografía adquiere mayor repercusión es en la apertura de las bocas eruptivas de los edificios volcánicos (figura 2). Sin embargo, muchos autores lo explican en relación con la dirección del viento dominante durante el paroxismo o con la inclinación del conducto eruptivo (Rittmann, 1963; Macdonald, 1972; Porter, 1972; Fúster et al., 1978; Derruau, 1991; Cas y Wright, 1987; Ollier, 1988; Inbar y Risso, 2001, etc.). Incluso se señala que tres son los factores principales que modifican su forma: «la energía de la erupción, el tipo y el tamaño de los piroclastos y la dirección y fuerza del viento durante la erupción» (Carracedo, 2006). Por tanto, es necesario valorar qué factores condicionan la apertura de los cráteres de estos volcanes en la isla de Tenerife y cuáles son los más importantes; y observar si puede extrapolarse a otras regiones volcánicas.

En Canarias, los análisis dedicados a este tipo de volcanes (Martínez de Pisón y Higes, 1971; Romero 1991 y 1992...) señalan que la forma que poseen no siempre se reduce a conos anulares y en herradura. Son tantos los factores que intervienen en su configuración morfológica —naturaleza y composición química del magma emitido, tipo y distribución de los materiales, tipo y disposición de las fracturas, estilo y evolución dinámica, intensidad del paro- 
xismo, duración del período activo, inclinación del conducto eruptivo, acción del viento, rasgos topográficos del área de asentamiento, situación hidrogeológica, etc. (Romero 1991 y 1992), así como las múltiples correlaciones posibles entre los mismos- que se puede afirmar que cada volcán muestra una morfología propia que lo diferencia del resto (Dóniz Páez, 2009).

Los volcanes basálticos monogénicos, al igual que en otras regiones volcánicas, son las formas más comunes producidas por el fenómeno volcánico subaéreo de Tenerife (Dóniz Páez, 2009 y 2010a). El interés que han despertado en la comunidad científica, a pesar de su elevado número, es menor que el de otras estructuras y procesos eruptivos. En el caso de Tenerife este hecho, sin duda, se debe tanto a su menor impronta paisajística, como a sus bajos índices de peligrosidad (Dóniz-Páez, 2010b). Sin embargo, la introducción de técnicas y herramientas específicas para su análisis (morfometría) y las potenciales repercusiones en la reactivación de sistemas volcánicos mucho más complejos (Teide-Pico Viejo) han determinado un aumento de los trabajos, fundamentalmente geomorfológicos referidos al estudio de los volcanes basálticos monogénicos de la isla de Tenerife (Dóniz Páez, 2009 y 2010a).

Dóniz Páez (2001) ya puso de manifiesto, de un modo aproximado, que la topografía condicionaba la forma final y el tamaño de los volcanes basálticos monogénicos. Por tanto, dada la diversidad y variedad de los factores que intervienen en la forma final de los conos, el objetivo de este trabajo es determinar qué influencia desempeñan los rasgos topográficos previos en la morfología final de los volcanes basálticos monogénicos anulares y en herradura, en términos de apertura o no de cráteres, y valorar su importancia relativa en relación con otros condicionantes.

\section{ÁREA DE ESTUdiO: GEOMORFOLOGía DE LOS VOLCANES}

Tenerife (Canarias, España) constituye la isla más extensa $\left(2034 \mathrm{~km}^{2}\right)$ y elevada del Archipiélago $(3718 \mathrm{~m})$ y posee enormes contrastes topográficos, desde ámbitos relativamente llanos hasta sectores abruptos y con pendientes elevadas. Esta isla se ha construido a partir de la acumulación de materiales volcánicos de diferente naturaleza (básica, ácida e intermedia) a lo largo de un dilatado período de tiempo que abarca varios millones de años. En Tenerife se reconocen diferentes tipos de edificios volcánicos (volcanes escudos, estratovolcanes, calderas, maares, anillos y conos de tobas, domos, conos de escorias, etc.); de todos ellos, por su número y extensión superficial, destacan los 297 volcanes basálticos monogénicos (figura 3). 
FIGURA 3

DISTRIBUCIÓN DE VOLCANES Y CAMPOS VOLCÁNICOS DE TENERIFE (DÓNIZ PÁEZ, 2005 MODIFICADO)

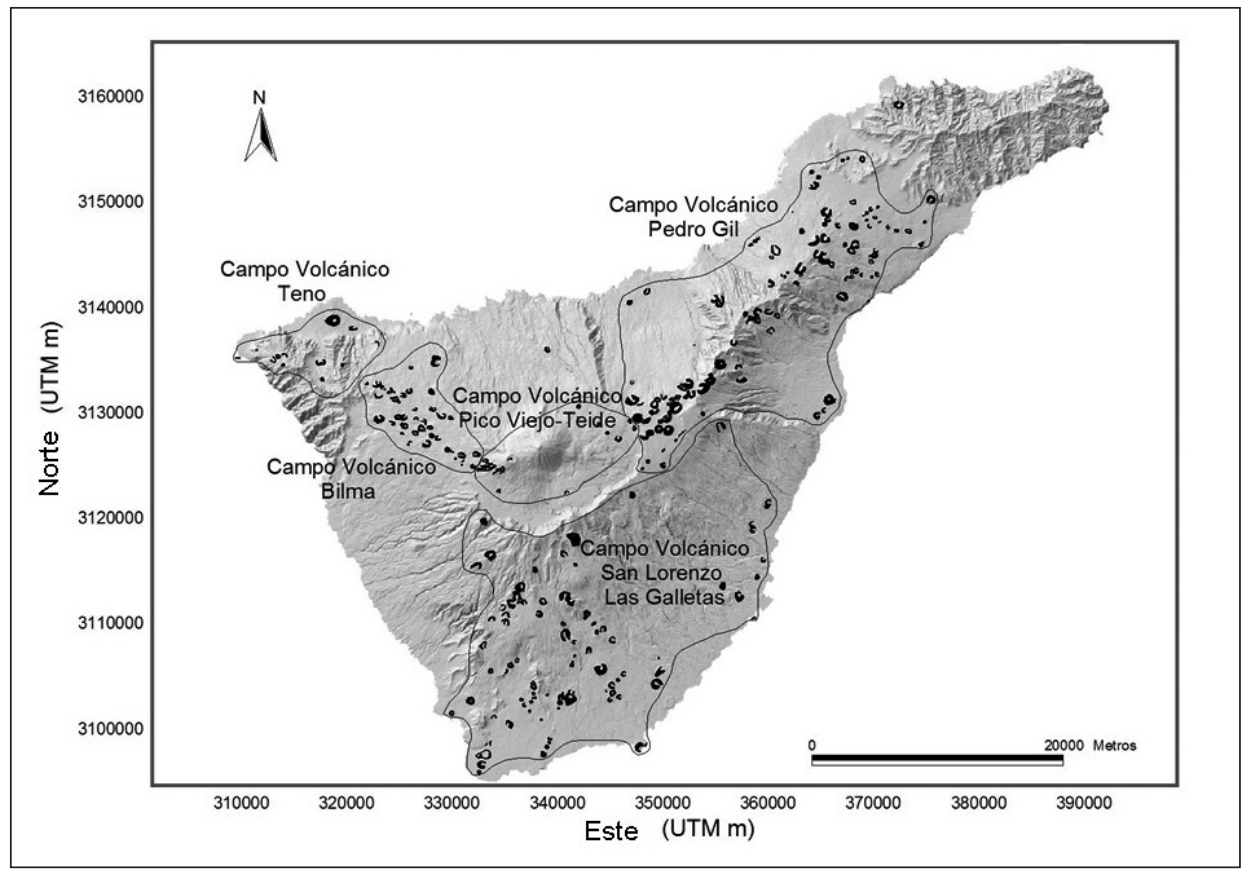

Estos edificios de escorias se agrupan en cinco campos volcánicos (figura 3) de diferente disposición geográfica (alineaciones, orientaciones, pendientes, etc.), pero con caracteres volcanológicos similares entre sí (Dóniz Páez, 2005); y en cuatro categorías morfológicas: edificios anulares cerrados, volcanes en herradura, aparatos múltiples y montañas de piroclastos. Dos de estos grupos se subdividen, a su vez, para obtener un total de siete subtipos morfológicos (Dóniz Páez et al., 2006). Los aparatos eruptivos monogénicos de Tenerife pueden, por tanto, estar abiertos o cerrados, y estar constituidos por un edificio o, por el contrario, por varios alargados, imbricados y yuxtapuestos con morfologías más o menos alargadas en sentido de la fractura y de la pendiente dominante. A su vez, la línea cratérica puede configurarse a partir de un solo cráter o varios, pudiendo estar abiertos o cerrados y con disposición circular o elongados siguiendo, del mismo modo, la dirección de la fractura y de la máxima pendiente. 
No todas las categorías morfoestructurales están igualmente representadas en el conjunto de Tenerife. Los conos en herradura son los más numerosos con 205 edificios (69,03\%), seguidos de los 39 edificios anulares cerrados $(13,14 \%)$, las acumulaciones de piroclastos con 34 (11,45\%) y por último los 19 volcanes múltiples $(6,38 \%)$. Estos datos ponen de manifiesto que las dos grandes categorías morfológicas definidas tradicionalmente (conos anulares y en herradura), suman más del $82 \%$ de los volcanes (Dóniz Páez, 2009); puesto que también en Tenerife constituyen las morfologías más habituales, es sobre estos dos subtipos donde se valorará qué influencia tiene la topografía en su forma.

Puesto que el estudio se centra solo en dos categorías morfológicas, a continuación se explica cada una de ellas.

\section{Geomorfología de los volcanes basálticos monogénicos de Tenerife}

\section{Los edificios volcánicos anulares}

El análisis morfológico de los conos anulares de Tenerife pone de manifiesto que estos edificios corresponden, habitualmente, a aparatos muy sencillos cuyos rasgos esenciales están en relación con el carácter más o menos circular de su planta y la existencia de cráteres cerrados (figura 4).

En diferentes modelos sobre la construcción de los volcanes simples (McGething et al., 1974; Dehn, 1987; Riedel et al., 2003), el carácter anular y la forma cerrada del cráter se explica siempre en función de tres factores principales:

1) el ángulo con que son expulsados los materiales piroclásticos,

2) la escasa dispersión de los productos de proyección aérea, y

3) la geometría del conducto eruptivo.

\section{Los edificios volcánicos en herradura}

Aparentemente los edificios en herradura muestran rasgos morfológicos muy simples, por lo que es habitual compararlos con los conos de tipo anular. Ahora bien, la diversidad morfológica de este tipo volcanes es, con diferencia, mucho más rica que la que presenta los conos anulares. Su carácter distintivo está en relación con que siempre carecen de una parte del dorso, por lo que sus cráteres están abiertos (figura 5). Poseen plantas circulares o alargadas 


\section{FIGURA 4}

\section{VOLCÁN ANULAR CERRADO DE MONTAÑA DE LA ATALAYA EN TACORONTE} AL NORTE DE TENERIFE

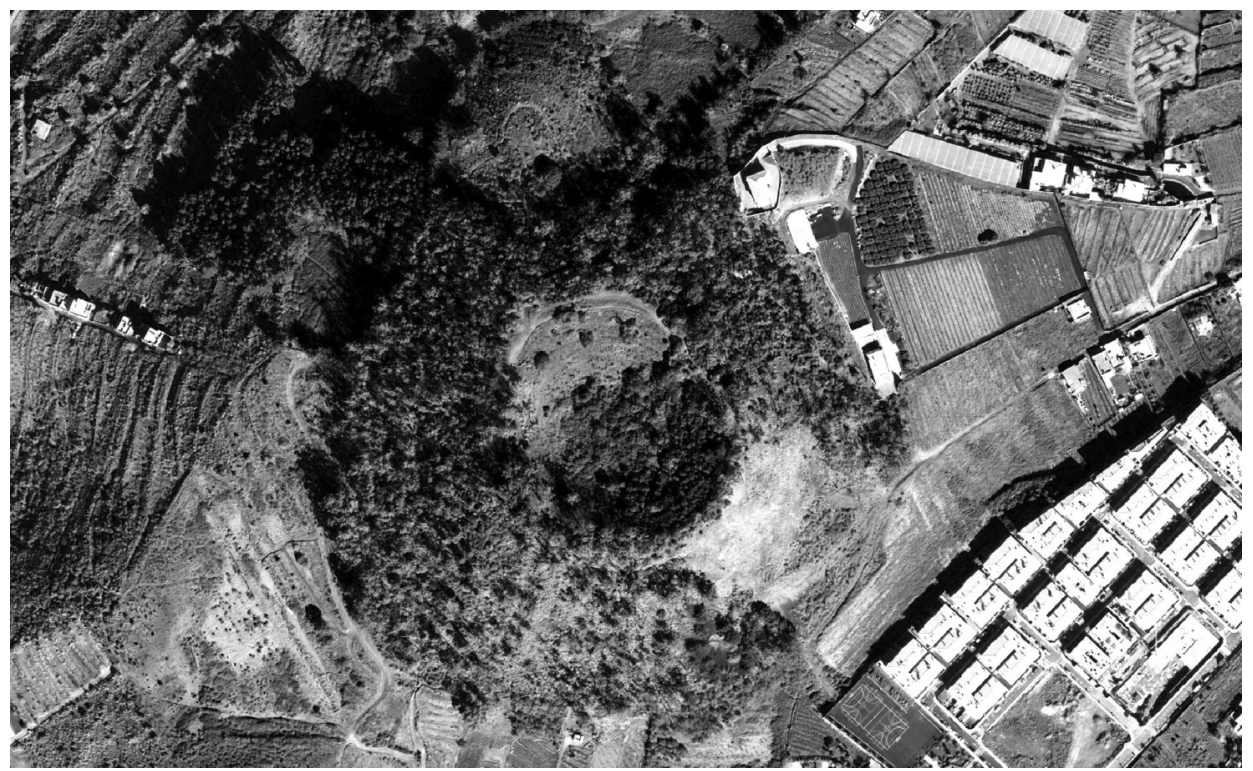

que están en relación con su construcción a partir de uno o más centros emisores. Dentro de este tipo de volcanes se pueden reconocer, a su vez, tres subcategorías: volcanes en herradura típica, de vértices prolongados o diapasón y en arco (Dóniz Páez et al., 2006).

La carencia de un sector del dorso del edificio puede ser consecuencia de su falta de construcción o de su destrucción como resultado de la emisión de coladas. Tanto en uno como en otro caso, el carácter abierto de los mismos está en relación con la actuación de varios factores:

1) el soplo del viento durante la erupción,

2) la pendiente del área de asentamiento del cono,

3) la dinámica eruptiva y

4) la inclinación del conducto eruptivo.

Evidentemente, cada uno de estos factores puede condicionar por sí solo la construcción de volcanes en herradura, aunque lo habitual es que actúen de forma combinada. De este modo, el soplo continuo del viento durante la erupción en una dirección puede determinar la acumulación de los productos de 
FIGURA 5

VOLCÁN ABIERTO EN HERRADURA DE MONTAÑA RASCA EN EL SUR DE TENERIFE

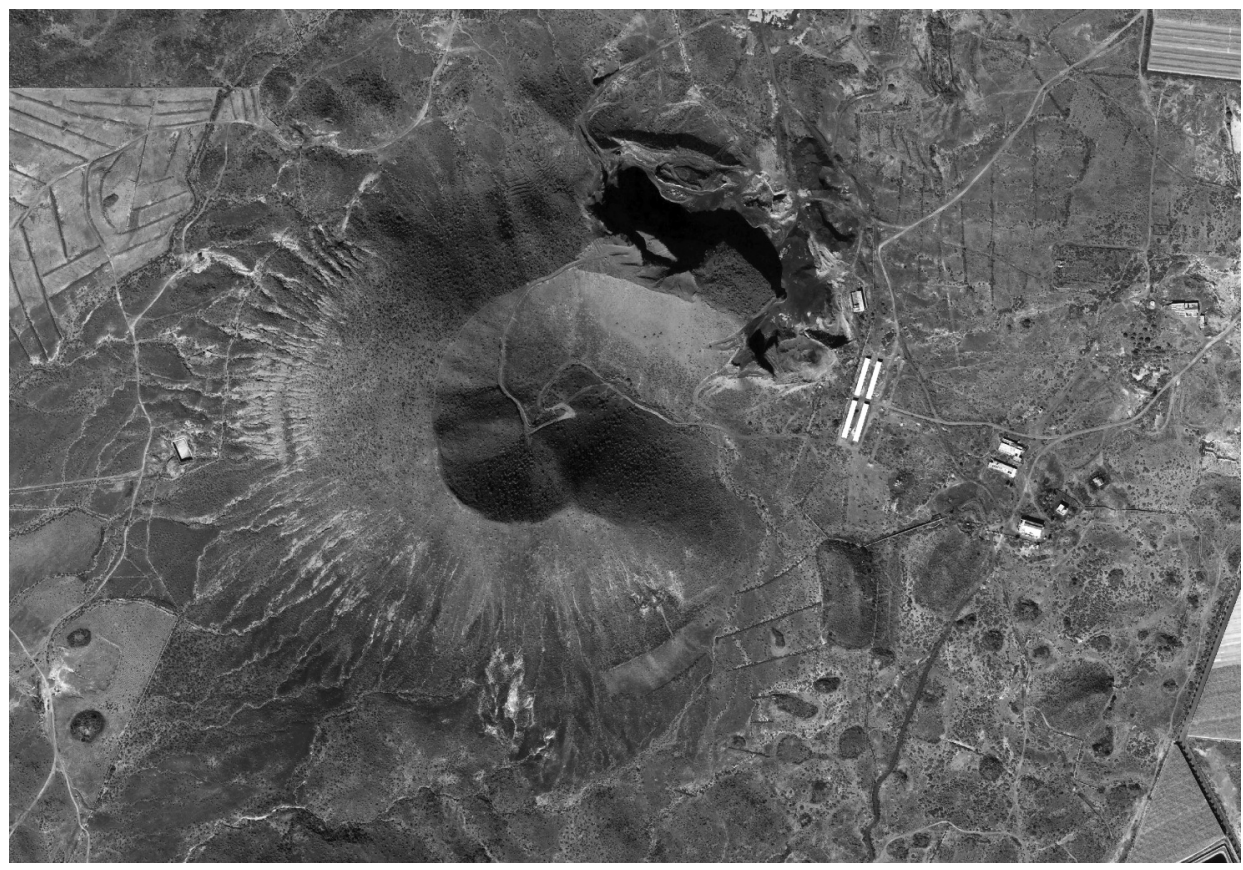

proyección aérea hacia el área de sotavento de los centros de emisión o de la fractura eruptiva, provocando un mayor crecimiento en altura de uno de los flancos del volcán respecto a los otros y, en ocasiones, impedir la construcción del flanco de barlovento y, por tanto, condicionar el derrame de las coladas de lava en sentido contrario al dorso del edificios de mayor altura (figura 5).

Por otro lado, bajo el efecto de la gravedad, las coladas de lava se derraman a favor de la pendiente, por lo que pueden llegar a inhibir la formación de uno de los flancos del edificio. A veces, el flujo de las coladas posee tasas de emisión tan altas que permiten su desbordamiento desde un cráter central, por lo que pueden llegar a desmantelar parcialmente el edificio. En este caso, fragmentos del aparato volcánico son incorporados y arrastrados en el seno del flujo lávico dando lugar a monolitos de escorias soldadas dispersos sobre las coladas («bloques erráticos»). 
También la dinámica eruptiva condiciona, en cierto modo, la morfología de los conos en herradura. Habitualmente la construcción de los conos de este tipo es resultado de una dinámica eruptiva moderada caracterizada, bien por la alternancia temporal de fases de distinto estilo - explosivas o efusivas-, bien por su concomitancia (fases mixtas explosivas-efusivas). Así, cuando existe alternancia de las fases eruptivas, la forma en herradura del cono se adquiere como consecuencia de la remodelación del dorso por el derrame de las coladas. Ahora bien, si la actividad es concomitante pueden generarse edificios que nunca hayan presentado morfologías cerradas, es decir, conos carentes desde el origen de uno de sus flancos.

Por último, los efectos ocasionados por la inclinación del conducto eruptivo en este tipo de erupciones son muy similares a los ejercidos por la acción del viento, de modo que el carácter en herradura de los edificios se adquiere como consecuencia de la acumulación preferente de los productos de proyección aérea hacia un sector determinado.

\section{Metodología}

Localizados, identificados y clasificados morfológicamente cada uno de los volcanes basálticos monogénicos de Tenerife a través de cartografía topográfica 1:10.000, fotografías aéreas 1:18.000, mapas geológicos 1:25.000 y, sobre todo, trabajo de campo (Dóniz Páez, 2009), se procedió a valorar, dentro del conjunto de factores señalados y que condicionan su forma, qué papel desempeña la topografía en la morfología final de los mismos, y si esta es determinante o no en la forma de este tipo de volcanes en Tenerife. En relación con este hecho, Tibaldi (1995), Tibaldi y Lagmay (2006) y Corazzato y Tibaldi (2006) concluyeron que para la rotura del cráter la pendiente regional domina sobre la tectónica, cuando la pendiente es superior a los $10^{\circ}$. Por tanto, este valor es el que se ha tenido en cuenta en este trabajo para valorar cuando un edificio está emplazado sobre pendientes llanas $\left(<10^{\circ}\right)$ o no $\left(>10^{\circ}\right)$.

Para ello, primero, se seleccionaron los 263 volcanes con cráteres, que suponen el 88,5\% del total de edificios de Tenerife. Segundo, se observó si poseen cráteres cerrados (39 volcanes anulares) o abiertos (205 volcanes en herradura), desechando del estudio los volcanes múltiples (19) que pueden disponer tanto de bocas eruptivas abiertas como cerradas y las montañas de lapilli que no disponen de cráteres. Tercero, si se emplazaban en sectores de topografía llanas con poca pendiente $\left(<10^{\circ}\right)$ o no $\left(>10^{\circ}\right)$. Cuarto, se correlacionó la apertura de los cráteres y la dirección de la máxima pendiente para cada uno de los 244 volcanes es- 
tudiados. Para los puntos tercero y cuarto se procedió a calcular la pendiente del área de emplazamiento de los volcanes a través de cartografía topográfica y trabajo de campo, y todo ello bajo un SIG (Arcview). Quinto, para los volcanes en herradura, se establecieron relaciones uno a uno para los 205 volcanes entre la dirección del punto culminante y la dirección-sentido de los vientos dominantes, con el fin de determinar su papel en la apertura de los cráteres.

Para este último paso, se llevó a cabo un análisis enfrentando los parámetros de dirección de apertura del cono con la dirección de la pendiente de emplazamiento de los mismos, encontrándose correlaciones elevadas entre los dos ítems. Es cierto que esto no significa, sin embargo, que la topografía sea el único factor que explica el carácter abierto de estos edificios volcánicos, aunque está claro que este parámetro constituye uno de los factores principales a tener en cuenta.

Para analizar cuál es la influencia que los vientos más frecuentes de la isla, los alisios, ejercen en la forma final de los edificios volcánicos en herradura, se llevó a cabo un estudio en el que se correlacionan los datos de dirección de apertura de los conos y los de orientación del punto culminante del aparato volcánico con la dirección de los vientos alisios, teniendo presente las variaciones en dirección que presentan estos vientos con la altura. Se establecieron tres intervalos altitudinales en función de la altitud media de la base de las inversiones térmicas de subsidencia en Tenerife, que son las más habituales, frente a las superficiales. Los intervalos son: $<800$ metros, entre 800-1500 metros y $>1500$ metros (Dorta, 1996).

Todos los registros de datos se llevaron a cabo de manera digital en una tabla de datos individualizada para cada conjunto eruptivo con más de 30 parámetros morfométricos, incluidos pendientes del área de emplazamiento de los volcanes, dirección de apertura de cráteres y dirección de punto culminante. Las diversas correlaciones se llevaron a cabo una a una para la totalidad de los 244 volcanes estudiados y se agruparon posteriormente (Excel) y se trabajaron bajo un SIG (Arcview).

\section{RESULTADOS}

\section{Relación topografía-volcanes basálticos monogénicos anulares}

Al analizar las características del emplazamiento de este tipo de volcanes se observa que 37 de los 39 edificios, que suponen el 95\% de los de su tipo, se localizan en ámbitos de rasgos topográficos de mínima pendiente (figura 4), lo que pone de manifiesto que la topografía del área de asentamiento de estos co- 
nos, en combinación con los otros factores mencionados, es uno de los condicionantes fundamentales que interviene en su morfología. Por tanto, para el 95\% de los aparatos eruptivos anulares la correlación entre el carácter cerrado de su cráter y el emplazamiento en sectores de escasos o mínimos desniveles es del $100 \%$. Es evidente que aunque prácticamente nunca se tiene en cuenta la topografía, si que es un condicionante importante en la configuración morfológica de volcanes anulares cerrados.

\section{Relación topografía-volcanes basálticos monogénicos en herradura}

Establecer si la apertura de los conos y de los cráteres de los edificios volcánicos en herradura se debe a uno solo de los factores mencionados o a la acción combinada de ellos no siempre resulta fácil. El análisis de los volcanes en herradura de Tenerife manifiesta la estrecha relación existente entre la morfología de los conos y su ubicación en áreas de pendientes siempre por encima de los $10^{\circ}$. De los 205 volcanes con cráteres abiertos, 195 edificios $(95,12 \%)$ se localizan en territorios con pendientes más o menos acusadas $\left(>10^{\circ}\right)$ y sólo diez $(4,88 \%)$ lo hacen en sectores de topografías llanas o poco contrastadas $\left(<10^{\circ}\right)$ (figura 5). Por tanto, para más del $95 \%$ de los casos la pendiente es la responsable directa de la rotura de sus bocas, mientras que el porcentaje restante se relaciona con otros factores como pueden ser la acción regular del viento, la geometría de los cráteres y la inclinación del conducto eruptivo.

Enfrentando las direcciones del punto culminante y la apertura del cráter con la dirección más frecuente de los vientos y con la altitud (figura 6) observamos que entre ellos no existe una buena correlación, de modo que los volcanes ubicados por debajo de los 800 metros de altitud poseen las orientaciones de los puntos culminantes y del sector de apertura del cráter independientemente del soplo de los alisios, que lo hace desde el primer cuadrante. Si estuvieran correlacionados, el punto culminante debería localizarse preferentemente en el tercer cuadrante y la apertura de las bocas eruptivas en el primero. Algo similar le sucede a los conos localizados por encima de los 1500 metros de altitud, donde la dirección más frecuente de los alisios es del tercer cuadrante y las orientaciones más habituales del punto culminante y la apertura del cráter son del tercer y primer cuadrante respectivamente. Para los aparatos volcánicos ubicados entre los 800-1500 metros de altitud, tanto el soplo de los alisios como las orientaciones del punto culminante y la apertura de los cráteres es muy variable, pero tampoco muestra correlaciones elevadas entre dirección del viento y punto culminante-apertura del cráter. 
FIGURA 6

ESQUEMA TEÓRICO EN EL QUE SE RELACIONA LA ALTITUD

Y LA DIRECCIÓN DOMINANTE DE LOS ALISIOS CON LA APERTURA

DEL CRÁTER Y ORIENTACIÓN DEL PUNTO CULMINANTE DE LOS VOLCANES

EN HERRADURA BASÁLTICOS MONOGÉNICOS DE TENERIFE

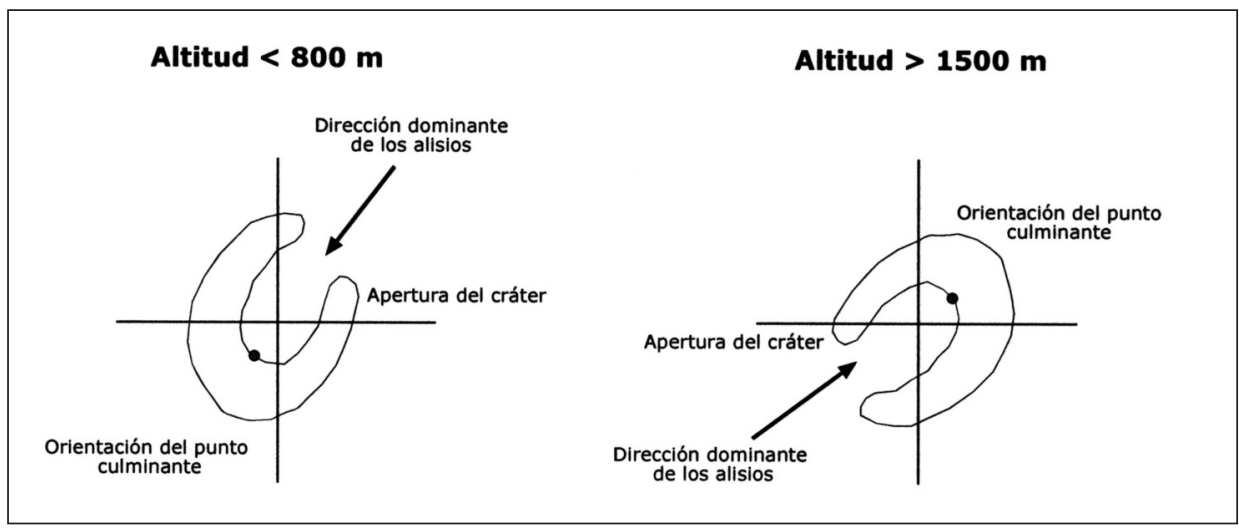

Todo esto pone de manifiesto que no existe una estrecha relación entre la apertura de los volcanes simples de Tenerife y la dirección dominante de los vientos más frecuentes de la isla y que, por tanto, la apertura de los mismos está más en relación con otros factores como la pendiente de las áreas de emplazamiento. Aun así, existen edificios eruptivos cuya dirección de apertura del cráter y localización del punto culminante están, como hemos señalado, acordes con el soplo dominante de los alisios a una altitud determinada, se trata siempre de volcanes emplazados fundamentalmente en sectores de escasa pendiente $\left(<10^{\circ}\right)$ y, sobre todo, en las proximidades del litoral.

\section{DISCUSIÓN Y CONCLUSIONES}

A pesar de que los trabajos dedicados a los volcanes basálticos monogénicos son cada vez más numerosos, no existen prácticamente estudios dedicados a establecer las relaciones entre su forma y los factores responsables de la misma. Sin embargo, si que hay publicaciones donde se enumeran cuáles son los principales causantes de la morfología final de este tipo de volcanes. En ellas se indica, tal y como se ha señalado, el papel fundamental del viento du- 
rante la erupción en la apertura de cráteres y de edificios. Por ello, es necesario que se realicen trabajos específicos dedicados a valorar la importancia relativa de cada uno de estos factores en la forma de los edificios eruptivos simples. En este sentido, en 2001, Dóniz Páez estableció, de manera aproximada, qué repercusiones desempeñaba la topografía en la morfología y en el tamaño de este tipo de aparatos eruptivos, pero sin valorar los efectos del viento y sin discriminar los conjuntos volcánicos múltiples.

Tenerife cuenta con 297 volcanes basálticos monogénicos, de los ellos, 263 poseen bocas eruptivas. La presencia de cráteres en los edificios volcánicos es el criterio fundamental para valorar el efecto de la topografía general en la apertura o no de los volcanes, tal y como han puesto de manifiesto Tibaldi (1995), Tibaldi y Lagmay (2006) y Corazzato y Tibaldi (2006). Por esta razón se desecharon las montañas de piroclastos sin cráter y los edificios múltiples que pueden disponer tanto de bocas abiertas como cerradas. En este sentido, el estudio se ha realizado sobre 244 aparatos eruptivos, que suponen el $82,15 \%$ de la muestra total; de ellos, 39 corresponde a conos volcánicos de forma anular y el resto a herradura.

De los 39 conos anulares, el 95\% (37 edificios) están emplazados en sectores de escasas o bajas pendientes $\left(<10^{\circ}\right)$, por lo que la correlación entre el carácter cerrado de sus cráteres y la topografía plana de las áreas de ubicación de este tipo de volcanes es del 100\%. Sólo para dos conjuntos eruptivos (5\% de los conos anulares) no existe tal relación. En estos casos, dado el carácter asimétrico de los aparatos volcánicos, el factor clave que tiende a explicar su configuración morfológica es la inclinación del conducto eruptivo (Riedel et al., 2003), y el viento durante el paroxismo, ya que coincide el sector más elevado del volcán con el del área de distribución de los piroclastos por efecto directo del soplo del viento.

De los 205 edificios en herradura, 195 conos (95,12\%) están emplazados en sectores con pendientes $>10^{\circ}$ y sólo 10 conos $(4,88 \%)$ lo hacen en áreas de escasas o nulas pendientes. Asimismo, con el fin de valorar si el viento es un factor decisivo o no en el carácter abierto de los cráteres y de los conos, se establecieron correlaciones entre la dirección predominante de los alisios a diferentes altitudes con la dirección de los puntos culminantes y con la apertura de los cráteres de los volcanes en herradura. En ambos casos se observó que la correlación era baja o nula. Este hecho, por tanto, confirma la idea del papel fundamental de la topografía de las áreas de emplazamiento de los volcanes en su morfología.

En este sentido, para los 10 conjuntos volcánicos en herradura se aprecia, al igual que para los dos aparatos eruptivos anulares, una total coincidencia 
entre los sectores más elevados del volcán y el área de distribución de los piroclastos por efecto directo del viento.

Todo esto pone de manifiesto que tanto la topografía previa donde se ubican los volcanes como el viento son dos factores a tener presentes en la morfología de los volcanes basálticos monogénicos. Ahora bien, tal y como se ha puesto en evidencia para más del $82 \%$ de los conos simples de Tenerife, el factor principal que condiciona la apertura o no de su cráteres es la topografía, y no el viento como tradicionalmente se ha dicho, coincidente con lo que han propuesto otros autores en otras regiones volcánicas (Tibaldi 1995, Corazzato y Tibaldi 2006). En este sentido, las elevadas correlaciones cráter cerrado/topografía llana para los volcanes de tipo anular y cráter abierto/topografía accidentada para volcanes en herradura parecen ponerlo de manifiesto.

Recibido: 15/07/2009

Aceptado: 22/07/2010

\section{BibLIOGRAFÍA}

Becerra-Ramírez, R. (2007): Aproximación al estudio de los volcanes de la región volcánica del Campo de Calatrava a través de las técnicas de análisis morfométrico, Trabajo de Investigación Doctorado, Inédito. Ciudad Real, UCLM.

Bertotto, G., Bjerg, E. y Cingolani, C. (2006): "Hawaiian and Strombolian style monogenetic volcanism in the extra-Andean domain of central-west Argentina". Journal of Volcanology and Geothermal Research, 158, pp. 430-444.

Calvari, S. y Pinkerton, H. (2004): "Birth, growth and morphologic evolution of the 'Laghetto' cinder cone during the 2001 Etna eruption". Journal of Volcanology and Geothermal Research, 132, pp. 225-239.

Corazzato, C. y Tibaldi, A. (2006): "Fracture control on type, morphology and distribution of parasitic volcanic cones: An example from Mt. Etna, Italy". Journal of Volcanology and Geothermal Research, 158, pp. 177-194.

Carracedo, J. (2006): Volcán Teide. Volcanología, interpretación de paisajes e itinerarios comentados. Santa Cruz de Tenerife, Cajacanarias.

Cas, R. y Wright, J. (1987): Volcanic Successions: Modern and ancient. London, Allen and Unwin.

Dehn, J. (1987): Model of cinder cone formation, M.Sc. Thesis. Tempe, AZ, Arizona, State University, $85 \mathrm{pp}$.

Derruau, M. (1991): Geomorfología. Barcelona, Ariel. 
Dóniz Páez, J. (2001): "La influencia de la topografía en las características morfológicas y morfométricas de los volcanes basálticos monogénicos de Tenerife (Islas Canarias)". Cuaternario y Geomorfología, 15 (3-4), pp. 125-129.

Dóniz Páez, J. (2005): "Los campos volcánicos basálticos monogénicos de la isla de Tenerife (Canarias, España)". Estudios Geográficos, 66 (259), pp. 461-480.

Dóniz Páez, J. (2009): Volcanes Basálticos monogénicos de Tenerife. Los Realejos, Concejalía de Medioambiente del Excmo. Ayuntamiento de los Realejos.

Dóniz Páez, J. (2010a): "Geomorfología de los volcanes basálticos monogénicos de la isla de Tenerife, Canarias”, en E. González Cárdenas, E. Escobar Lahoz, R. Becerra Ramírez. R. Gosálvez Rey y J. Dóniz Páez (eds.), Aportaciones recientes en volcanología 2005-2008. Ciudad Real, Centro de Estudios Calatravos, pp. 181-203.

Dóniz Páez, J. (2010b): "El volcanismo basáltico monogénico de Tenerife (Canarias, España): reflexiones sobre el peligro y el riesgo". Scripta Nova. Revista Electrónica de Geografía y Ciencias Sociales [en línea]. Barcelona, Universidad de Barcelona, 20 de mayo de 2010, XIV, 324, http://www.ub.es/geocrit/sn/sn-324.htm (consultado 25/02/2011).

Dóniz Páez, J., Romero, C., Coello, E., Guillén, C. y García-Cacho, L. (2006): "El uso de técnicas morfométricas para la clasificación geomorfológica de los volcanes basálticos monogénicos de la isla de Tenerife (Canarias, España)", en A. Pérez Alberti. y J. López Bedoya (eds), Geomorfología y territorio. Santiago de Compostela, Universidad de Santiago de Compostela, pp. 723-739.

Dóniz Páez, J., Romero, C., Coello, E., Guillén, C., Sánchez, N., García-Cacho, L. y García. A. (2008): "Morphological and statistical characterisation of recent mafic volcanism on Tenerife (Canary Islands, Spain)". Journal of Volcanology and Geothermal Research, 173 (3-4), pp. 185-195.

Dorta, P. (1996): "Las inversiones térmicas en Canarias". Investigaciones Geográficas, 15, pp. 109-124.

Fúster, J., Araña, V., Brandle, J., Navarro, M., Alonso, U. y Aparicio A. (1968): Tenerife. Madrid, Instituto Lucas Mallada-CSIC.

González Cárdenas, E., Gosálvez Rey, R. U. y Escobar Lahoz, E. (2006): Volcanes del Campo de Calatrava; Guía divulgativa. Ciudad Real, Asociación para el Desarrollo del Campo de Calatrava.

Inbar, M. y Risso, C. (2001): "A morphological and morphometric analysis of high cinder cone volcanic field - Payun Matru, south-central Andes-Argentina". Zeitschrift fur Geomorphologie, 45 (3), pp. 321-344.

Macdonald, G. (1972): Volcanoes. Prentice-Hall, Englewood Cliffs.

Macgethin, T., Settle, M., Chouet, B. (1974): "Cinder cone growth modeled after northeast crater, Mount Etna, Sicily". Journal of Geophysical Research, 79 (23), pp. 3257 3272 .

Martin, U. y Németh, K. (2006): "How Strombolian is a 'Strombolian' scoria cone? Some irregularities in scoria cone architecture from the Transmexican Volcanic 
Belt, near Volcán Ceboruco, (Mexico) and Al Haruj (Libya)". Journal of Volcanology and Geothermal Research, 155, pp. 104-118.

Martínez de Pisón, E. y Higes Rolando, V. (1971): "La escala del tiempo en la evolución geomorfológica del aparato eruptivo del Teneguía”. Estudios Geográficos, 127, pp. 363-378.

Ollier, C. (1988): Volcanoes. Oxford, Blackwell.

Pallí, L y Pujadas, A. (1999): "El campo volcánico catalán". Enseñanzas de la Tierra, 7.3, pp. 229-236.

Poblete, M. (1994): El relieve volcánico del campo de Calatrava (Ciudad Real). Oviedo, Junta de Comunidades de Castilla La Mancha-Universidad de Oviedo.

Porter, S. (1972): "Distribution, morphology, and size frequency of cinder cones on Mauna Kea volcano, Hawaii”. Geol. Soc. Am. Bull., 83, pp. 3607-3612.

Riedel, C., Ernst, G. y Riley, M. (2003): "Controls on the growth and geometry of pyroclastic constructs". Journal of Volcanology and Geothermal Research, 127, pp. 121-152.

Rittman, A. (1963): Les volcans et leur activité. Paris, Masson.

Romero, C. (1991): Las manifestaciones volcánicas históricas del Archipiélago Canario. Santa Cruz de Tenerife, Consejería de Política Territorial, Gobierno Autónomo de Canarias.

Romero, C. (1992): Estudio geomorfológico de los volcanes históricos de Tenerife. Santa Cruz de Tenerife, ACT.

Takada, A. (1994): "The influence of regional stress and magmatic input on styles of monogenetic and polygenetic volcanism". Journal of Geophysical Research, 99 (B7), pp. 13563-13573.

Tibaldi A. (1995): "Morphology of pyroclastic cones and tectonics". Journal of Geophysical Research, 100 (B12), pp. 24521-24535.

Tibaldi A. y Lagmay, A. (2006): "Interaction between volcanoes and their basements". Journal of Volcanology and Geothermal Research, 158, pp. 1-5.

Valentine, G. y Keating, G. (2007): "Eruptive styles and inferences about plumbing systems at Hidden Cone and Little Black Peak scoria cone volcanoes (Nevada, U.S.A.)". Bull. Volcanol., 70, pp. 105-113.

Valentine, G., Krier, D., Perry, F. y Heinken, G. (2007): "Eruptive and geomorphic processes at the Lathrop Wells scoria cone volcano". Journal of Volcanology and Geothermal Research, 161, pp. 57-80.

Vespermann, D. y Schmincke, H. (2000): "Scoria cones and tuff rings", en Sigurdsson, H., Houghton, B., Rymer, H., Stix, J. y Mcnutt, S. (eds.), Encyclopedia of volcanoes. San Diego, Academic Press, pp. 683-694.

Wood, C. (1980a): "Morphometric evolution of cinder cones". Journal of Volcanology and Geothermal Research, 7, pp. 387-413.

Wood, C. (1980b): "Morphometric analysis of cinder cone degradation". Journal of Volcanology and Geothermal Research, 8, pp. 137-160. 


\section{RESUMEN}

Este trabajo analiza las relaciones entre la topografía previa y la morfología de los volcanes basálticos monogénicos de Tenerife. Se establecen las correlaciones entre el emplazamiento topográfico y la apertura o no de los cráteres de los 39 volcanes anulares y de los 205 volcanes en herradura. Se observa que en ambos casos existen elevadas correlaciones del orden de 0,95. El 95\% de los volcanes anulares están emplazados en áreas de topografía plana $\left(<10^{\circ}\right)$, mientras que el $95 \%$ de los volcanes abiertos en herradura se ubican en áreas de topografía más accidentada. Tradicionalmente la topografía ha sido considerada como un factor secundario en la forma de los volcanes; sin embargo, según estos datos, es un factor clave que controla la morfología de los volcanes basálticos monogénicos.

PALAbRaS ClaVE: volcanismo basáltico monogénico; topografía; morfometría; Tenerife; Canarias.

\section{AbSTRaCt}

Relationship between topography and morphology of cinder cones of Tenerife (Canary Islands, Spain). This paper analizes the set of relations between previous topography and the morphology of basaltic monogenic volcanoes on Tenerife. It establishes relations between the topographical setting and the (lack of ) opening of craters in the 39 ring volcanoes and the 205 horshoe-shaped volcanoes. In both cases, there is a high ratio of correlations (up to 0.95 ). Some $95 \%$ of the ringed volcanoes are located in flat topographical zones $\left(<10^{\circ}\right)$, whereas some $95 \%$ of those open in a horshoe form appear in more abrupt areas. Traditionally, topography has been disregarded as a secondary factor in the shape of volcanoes, but, according to these data, it is a key element that determines the morphology of basaltic monogenic volcanoes.

KEY WORDS: cinder or scoria cone; topography; morphometric; Tenerife; Canary Islands.

\section{RÉSUMÉ}

Liens entre topographie et morphologie des volcans basaltiques monogéniques. Ce travail analyse les liens entre la topographie préalable et la morphologie des volcans basaltiques monogéniques de Tenerife. On peut établir des relations entre l'emplacement topographique et l'ouverture ou non des cratères des 39 volcans en forme d'anneau et des 205 en forme de fer à cheval. On peut observer qu'il existe dans les deux cas des corrélations élevées de l'ordre de 0,95. 95\% des volcans en forme d'anneau sont situés dans des zones topographiques planes $\left(<10^{\circ}\right)$, tandis que $95 \%$ des volcans ouverts en forme de fer à cheval se trouvent dans des zones topographiques plus accidentées. Traditionnellement, la topographie a été considérée un facteur secondaire dans la forme des volcans. Pourtant, selon ces données, il s'agit d'un facteur clé qui contrôle la morphologie des volcans basaltiques monogéniques.

Most CLÉs: Cônes de scories; Topographie; morphométrie; Tenerife; Îles Canaries. 